$>$ Le cadre nosologique des myopathies inflammatoires (MI) a évolué. L'objectif était de comparer la répartition de ces $\mathrm{Ml}$ entre diagnostic initial et après relecture avec application des classifications récentes. Ont été inclus les 28 patients suivis à Bordeaux avec un diagnostic de Ml, ayant eu une biopsie et un bilan entre 2012 et 2014 . Parmi eux, $18 \%$ avaient un auto-anticorps spécifique. Tous ont été traités par corticoïdes, plus de $3 / 4$ par immunosuppresseurs. Initialement on retrouve une majorité de polymyosite ( $P M$ ) et dermatomyosite (DM). Après relecture, $39 \%$ sont classés comme myosite de chevauchement (MC), $25 \%$ comme DM et la part des myopathies nécrosantes auto-immunes (MNAI) augmente à $18 \%$. Sous réserve d'un travail rétrospectif, les données concordent avec la littérature: haute prévalence des MC, sousestimation des MNAI, intérêt d'individualiser les groupes de patients de moins bon pronostic. <

Le cadre nosologique des MI connaît d'importantes évolutions avec l'individualisation d'entités comme les MNAl ou le syndrome des anti-synthétases (AS). La découverte de nouveaux auto-anticorps (AAC) et l'amélioration des techniques histologiques participent à une meilleure connaissance des MI. Une nouvelle classification a été proposée [1] avec un intérêt diagnostique mais aussi pronostique et thérapeutique [2]. Cliniquement les MI se présentent par un déficit moteur proximal subaigu avec myalgies. Les troubles de la déglutition et respiratoires ne sont pas rares. Le diagnostic repose sur la clinique, l'élévation franche des CK, éventuellement l'imagerie mais surtout sur la réalisation d'une biopsie musculaire (BM).

Longtemps on a distingué les dermatomyosites (DM) et les polymyosites (PM). Cette classification a été remise en cause par la découverte de nouveaux $A A C$ et le démembrement d'entités spécifiques, la PM «isolée 》 est devenue rare [3]. II est désormais consensuel de classer les MI au sein des entités suivantes: dermato-

\section{Diagnostic \\ des myopathies \\ inflammatoires au \\ CHU de Bordeaux \\ de 2012 à 2014 \\ Application des nouvelles \\ classifications}

\section{Fanny Duval}

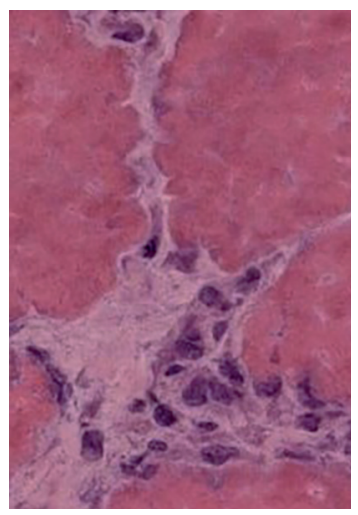

Service de neurologie, centre de référence des maladies neuromusculaires, hôpital Pellegrin, CHU, Bordeaux, France. fanny.duval@chu-bordeaux.fr

myosites; myosites de chevauchement [4] classées en trois entités: associées à une connectivite, associées à un $A A C$ non spécifique, syndrome des AS [5]; PM (définition histologique et absence d'AAC associé ou spécifique) et MNAI [6,7].

\section{Méthodes}

L'objectif de ce travail monocentrique rétrospectif était d'appliquer les classifications les plus récentes, aux patients suivis au CHU de Bordeaux entre 2012 et 2014 avec un diagnostic de MI primitive, en reprenant l'ensemble du dossier (clinique, biologique, histologique...) et de comparer l'évolution entre le diagnostic retenu initialement à celui retenu après relecture de l'ensemble du dossier. Les myosites à inclusions (IBM) sont exclues de ce travail.

Etaient donc inclus les patients adultes biopsiés entre le 01/01/2012 et le 31/12/2014 avec un diagnostic initial de Ml et ayant eu un bilan immunologique étendu. Ont été exclus de ce travail les patients ayant eu un bilan biologique incomplet ou suivis moins de trois mois ainsi que les patients biopsiés mais non suivis sur le CHU, et ceux dont le suivi a permis de retenir un diagnostic autre (IBM, hémopathie, myopathie infectieuse)

Ont été recueillis à partir du dossier informatisé : âge, sexe, service d'origine et présentation clinique, taux de CK initial, résultats du bilan immunologique, résultats de la BM ainsi que les traitements et l'évolution. Pour les dossiers discordants (entre diagnostic initial ana- 


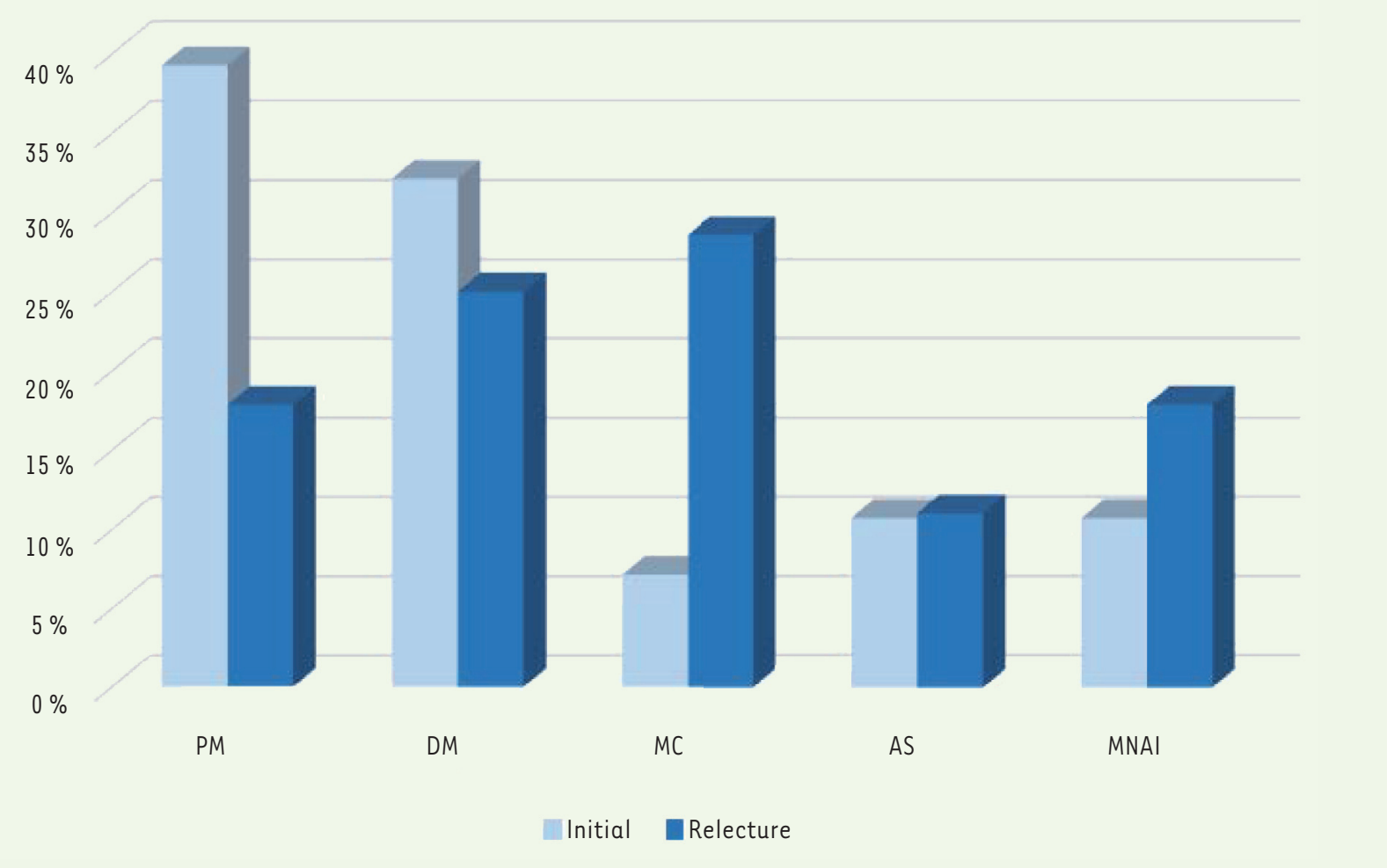

Figure 1. Évolution du diagnostic. PM : polymyosite; DM : dermatomyosite ; MC : myosite de chevauchement hors syndrome des anti-synthétases ; AS : syndrome des anti-synthétases; MNAI : myopathie nécrosante à médiation auto immune.

tomopathologique et celui du clinicien), une relecture a été réalisée par le Pr Martin-Négrier, avec si nécessaire la réalisation de techniques complémentaires non disponibles initialement. L'ensemble des dossiers clinico-biologiques et histologiques a ensuite été rediscuté. Les critères utilisés sont ceux s'appuyant sur les classifications de l'ENMC international workshop de 2003 [8] et une publication concernant plus spécifiquement les myosites de chevauchement [9].

Sur les 83 dossiers remplissant les critères sur la période étudiée, 22 ont été exclus (absence de suivi sur le CHU), trois par manque de données, 30 du fait d'un diagnostic final retenu discordant (principalement IBM mais aussi de manière plus anecdotique SLA, GVH musculaire...).

Au final 28 dossiers remplissaient l'ensemble des critères dont une dizaine a nécessité une relecture par l'anatomopathologiste.

\section{Résultats}

Dans cette série, on note $85 \%$ de sujets féminins, l'âge moyen diffère peu entre les deux sexes. Une très large majorité est issue des services de neurologie, de médecine interne et de dermatologie (Figure 1).

$85 \%$ des patients avaient un déficit moteur, $15 \%$ des myalgies isolées ou une découverte fortuite d'élévation des CK dans un bilan de connectivite.

$25 \%$ avaient une atteinte distale associée et $21 \%$ une atteinte axiale. La moitié avait une atteinte cutanée (uniquement des DM et MC).
$21 \%$ avaient une atteinte respiratoire (3 AS, 1 DM, des MC). Le taux moyen de CK était de $3262 \mathrm{U} / \mathrm{L}$ (avec des valeurs très variées: de 43 à $16500 \mathrm{U} / \mathrm{L}$ ). La présence d'un anticorps anti-antigènes nucléaires solubles était détectée chez $35 \%$ (surtout des anti-SSA). Le panel «dot-myosite » n'apportait une réponse positive que dans $18 \%$ des cas ( 3 anti Mi2, 2 anti-PL7). Une imagerie musculaire a été réalisée chez $61 \%$, (IRM pour $81 \%$ des imageries réalisées) avec des signes évocateurs de MI dans $92 \%$ des cas. $100 \%$ des patients ont reçu une corticothérapie après le diagnostic, $79 \%$ des patients ont reçu au moins un immunosuppresseur, dont 4 ont reçu un traitement par cyclophosphamide. Les IgIV ont été utilisées chez $42 \%$, en phase initiale ou lors de rechute au sevrage des corticoïdes.

\section{Discussion}

Sur les diagnostics initiaux, on notait une nette prédominance PM suivies par les DM. L'application des critères les plus récents entraîne mécaniquement l'augmentation de la proportion des MC (tous types confondus) qui est la plus représentée, suivie par les DM. Cet élément est concordant avec les séries rapportées dans la littérature [1]. II reste 5 cas étiquetés PM, qui ne 
présentent pas de signes cutanés, pas de stigmates d'auto-immunité sur le plan biologique, pas d'argument histologique ou évolutif pour un autre diagnostic. Le taux de patients ayant une positivité pour un AAC paraît faible comparé aux chiffres avancés jusqu'à $80 \%$ de positivité dans certaines séries [10]. Les titres les plus élevés pour les AAC non spécifiques se retrouvent dans le cadre des MC. Se pose donc la question de la sensibilité des techniques immunologiques utilisées et de la recherche exhaustive de signes en faveur d'une connectivite chez ces patients, ce taux de PM restant supérieur à ceux avancés dans les articles les plus récents.

La relecture de certaines biopsies et la réalisation de techniques complémentaires en immuno-histochimie (C5-b9...) a permis d'affiner ou de corriger certains diagnostics sur le plan histologique. Néanmoins aucune de ces techniques n'est totalement sensible [11, 12] et la relecture des dossiers montre que tous les critères histologiques ne sont pas présents, même avec des tableaux cliniques typiques. Chez plusieurs patients, le marquage du CMH de classe I était peu évocateur d'une myopathie inflammatoire alors que le reste du tableau l'était. Cela illustre la nécessité d'utiliser des techniques complémentaires et du dialogue anatomopathologiste/cliniciens.

De par son caractère rétrospectif et des critères d'exclusions stricts sur le plan de la complétude des données clinico-biologiques il existe un probable biais de sélection avec probable sous-estimation de la catégorie MNAI et des anti-synthétases. On peut aussi s'interroger sur la sous-représentation des formes sévères ou paranéoplasiques dans cette série, là aussi par un possible biais de recrutement.

Ce travail illustre les difficultés diagnostiques parfois posées par les MI. La plupart des caractéristiques des patients de cette série est concordante avec les données de la littérature, néanmoins du fait d'un probable biais de sélection, elle en diffère par une moindre sévérité.

\section{En conclusion}

L'application des classifications les plus récentes aboutit à une répartition différente des patients avec une diminution $P M$ et une plus grande fréquence $M C$, ce qui est concordant avec les données de la littérature. La meilleure connaissance des spécificités de certains syndromes comme ceux des anti-synthétases ou les MNAI, tant sur le plan clinique que biologique et histologique apportera probablement encore des modifications à cette répartition au cours des années à venir. L'intérêt principal reste à aboutir à des groupes plus homogènes de patients ayant des caractéristiques évolutives spécifiques, afin d'en optimiser le suivi et le traitement. La discussion du dossier clinique et biologique avec l'anatomopathologiste paraît donc primordiale dans cette optique. L'utilisation en routine de nouveaux immunomarquages apporte des éléments supplémentaires, de même que l'élargissement des panels sanguins d'auto-anticorps détectés. Cependant le diagnostic reste posé sur un faisceau d'arguments, aucune technique n'étant totalement sensible et spécifique. L'utilisation de bases de données dédiées alimentées de manière prospective permettrait probablement une meilleure connaissance de la répartition, de l'incidence et du profil évolutif de ces patients.
Diagnosis of inflammatory myopathies at the CHU Bordeaux from 2012 to 2014: implementation of novel classifications

\section{LIENS D'INTÉRÊT}

Les auteurs déclarent n'avoir aucun lien d'intérêt concernant les données publiées dans cet article.

\section{RÉFÉRENCES}

1. Benveniste 0 . Acquired inflammatory myopathies: interest of specific autoantibodies for their classification. Rev Prat 2015 ; 65 : 607-12.

2. Dalakas MC. Inflammatory muscle diseases: a critical review on pathogenesis and therapies. Curr Opin Pharmacol $2010 ; 10: 346-52$.

3. Amato AA, Griggs RC. Unicorns, dragons, polymyositis, and other mythological beasts. Neurology $2003 ; 61: 288-9$.

4. Allenbach $y$, Benveniste 0 . Auto-anticorps au cours des myosites. Rev Med Interne $2014 ; 35: 437-43$.

5. Troyanov Y, Targoff IN, Payette MP, et al. Redefining dermatomyositis: a description of new diagnostic criteria that differentiate pure dermatomyositis from overlap myositis with dermatomyositis features. Medicine (Baltimore) $2014 ; 93: 318-32$.

6. Mahler M, Miller FW, Fritzler MJ. Idiopathic inflammatory myopathies and the anti-synthetase syndrome: a comprehensive review. Autoimmun Rev 2014 ; $13: 367-71$.

7. Stenzel W, Goebel HH, Aronica $\varepsilon$. Review: immune-mediated necrotizing myopathies: a heterogeneous group of diseases with specific myopathological features. Neuropathol Appl Neurobiol 2012 ; 38 : 632-46.

8. Allenbach Y, Drouot L, Rigolet A, et al. Anti-HMGCR autoantibodies in European patients with autoimmune necrotizing myopathies: inconstant exposure to statin. Medicine (Baltimore) $2014 ; 93: 150-7$.

9. Hoogendijk JE, Amato AA, Lecky BR, et al. $119^{\text {th }}$ ENMC international workshop: trial design in adult idiopathic inflammatory myopathies, with the exception of inclusion body myositis, 10-12 0ctober 2003, Naarden, The Netherlands. Neuromuscul Disord $2004 ; 14$ : 337-45.

10. Troyanov Y, Targoff IN, Tremblay JL, et al. Novel classification of idiopathic inflammatory myopathies based on overlap syndrome features and autoantibodies: analysis of 100 French Canadian patients. Medicine (Baltimore) $2005 ; 84: 231-49$.

11. Mammen AL. Autoimmune myopathies: autoantibodies, phenotypes and pathogenesis. Nat Rev Neurol 2011 ; 7 : 343-54

12. Van De Vlekkert J, Maas M, Hoogendijk JE, et al. Combining MRI and muscle biopsy improves diagnostic accuracy in subacute-onset idiopathic inflammatory myopathy. Muscle Nerve $2015 ; 51: 253-8$.

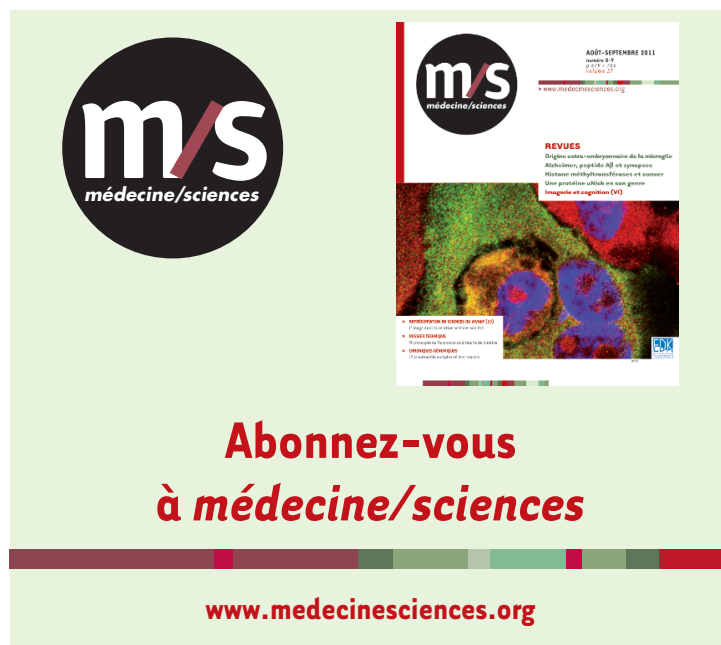

TIRÉS À PART

F. Duval 